\title{
Understanding Health Communication: Trends and Possibilities
}

\author{
Sudhansubala Sahu* and Preethi N R**
}

\begin{abstract}
Health communication is a branch of communication studies that deals with the communication patterns and practices adopted in health education discourses. All forms of communicationare oriented towards promoting information related to health falls under the domain of Health Communication. In this context, the present study attempts to assess existing trends of the health communication in India with special reference to research done in rural India. It highlights views on how health communication can be more effective if it is provided with appropriate resources. It deliberates on few possibilities that can be explored to make it more effective in future, especially in the era of Information Communication and Technology. It highlights many significant ruptures in research in the contemporary scenario, especially with respect to India and argues for some highly significant and interesting study to analyze how the latest innovations in ICT have influenced the dynamics between the three stakeholders of healthcare.
\end{abstract}

* Department of Sociology, Christ University, Bengaluru, India sudhansubala.sahu@christuniversity.in

** Department of Sociology, Christ University, Bengaluru, India preethi.nr@christuniversity.in 
Keywords: Health Communication, Health and Media, health education discourses, Health awareness, Privatization of health care, ICTs

\section{Introduction}

The year 2011 marked the start of the last five year period of the Millennium Development Goals (MDGs). What is more interesting from the point of view of this study is six out of the eight MDGs focus on or are related to health. Huge investments are being madein health communication both from the National and International communities to address these MDGs. Health communication is a branch of communication studies which deals with the communication patterns and practices used in campaigns, health education discourses, awareness programmes and also the simple conversation happening between the two major actors in the field of health Doctor and his patient. All forms of communicationare oriented towards promoting information related to health falls under the domain of Health Communication. In this context, the present study attempts to assess existing trends of the health communication in India with special reference to rural India and few possibilities that can be explored to make it more effective in future, especially in the era of Information Communication and Technology. This is done through are view of the literature, which is part of a working paper and also contributes to an ongoing project funded by ICSSR.

The relation between mass media and health has been immense. The purpose of the present study is to see how scholars have argued about making it more intense by re-strategizing the campaign with the help of health care providers. To add to this, they have also focused on the importance of delivering these messages to the target audience in a proper environment and ensuring maximum viewership for such campaigns. It also highlights views on how health communication can be more effective (especially in developing countries) if it is provided with appropriate resources. Along with this it briefly emphasizes the importance of new information communication technologies, especially the Apps in the field of Health Communication. 


\section{Communication and Public Health}

Communication in the field of health can be understood as an inevitable part of the system. What marks the difference is that such communications and information were considered - both by the public as well as the policy makers - as common sense. As we know, such information or knowledge did not require a planned approach and intervention to be carried out.In fact,health-related communication was understood as a natural process. Until the emergence of modern medicine, communication was largely informal. Those who practiced folk medicine communicated the natural ingredient's usage etc. through primary means of communication. This was followed by a pattern called intergenerational communication where critical information was passed on to the immediate heirs within the clan. Then came the phase where medicine was taken as a discourse and people were certified as doctors, and it was in this phase where the faith on doctor's words turned high. During this period communication between the doctor and the patient was a greater healer than the medicine itself. Therefore doctors with greater communication skills were sought after the most, considering their medical techniques of healing to be much more effective (Dawodu, 2007).

The first organized step to communication in the field of health was laid when the International Association for Communication (ICA) was incorporated in the year 1950. ICA was more of an academic union which conducted research in the field of communication and applied it in all aspects of health and humanity. Considering the significance of health based communication this association expanded a separate wing known as the Health Communication Division which worked with the sole objective of promoting and improving the patient- doctor communication techniques. This was a key stepping stone for a full- fledged study on health communication. Several researches conducted in these lines suggested that improvement in the verbal and non-verbal communication between the doctor and the patient had a direct impact on the health outcome and level of job satisfaction of these two actors. 
The next significant step following this was introducing health communication as a subject in schools and universities. This strengthened the ground of health and communication-based studies. Given the resources various campaigns and health promotional activities which followed then were found to be taken up in a more organized way. Several sovereign institutions like the United Nations, World Health Organization, and Centre for Disease Control and Prevention institutions have already noticed the significance of health communication establishing a special place in their respective institutions constructing the purpose of enhancing communication to mitigate health risks as well as improving the overall health status.

Having discussed all these positive and progressive aspects of Health Communication, we all must agree that it is often stuck in a vicious circle. Firstly, because it is heldin low esteem it is not given sufficient funding for rigorous evaluation, and hence the field does not progress. Since it does not progress, it is held in low esteem (Fox 2012: 58). At the same occasion, as a field of inquiry, Health communication does not allow much space for innovation and risk taking because it needs to respond to broad public support and also demands results. The enormous pressure on the health organizations including Government to show result does not allow time or space for are thinking of health communication thus reaching automatically for the current thinking, the existing models, and the usual ways of doing things (Fox, 2012, 53). Most of the health communications models rarely become a subject of review because the funding agencies hardly ever set the bar very high on measuring actual changes in behavior due to the exposure to health communication. But without sufficient data on actually sustainable changes in knowledge and behavior, it becomes hard to know what works and what does not (ibid.: 54).

Marsden \& Peter Freund argued that adoption of marketing principles would provide public health departments with a guide and incentive to help the practitioners as well as patients (cited in Tones \& Tilford, 2010, 347). But the fundamental difference that exists between commercial products and health is the reason why the goal is yet to be achieved. Both Atkin \& Wallack (1990) and Tones \& Tilford (2010) have clearly outlined these complexities and 
conflict in the objectives of these two institutions. Atkin \& Wallack (1990) summarize the conflicting priorities of mass media versus public health institutions in the following manner:

Table 1

\begin{tabular}{|l|l|}
\hline \multicolumn{1}{|c|}{ Mass Media Objectives } & \multicolumn{1}{c|}{ Public Health Objectives } \\
\hline To entertain, persuade or inform & To educate \\
\hline To make profit & To improve public health \\
\hline To reflect society & To change society \\
\hline To address personal concern & To address societal concerns \\
\hline To cover short-term events & To conduct long-term campaigns \\
\hline $\begin{array}{l}\text { To deliver salient pieces of } \\
\text { material }\end{array}$ & $\begin{array}{l}\text { To create understanding of } \\
\text { complex information }\end{array}$ \\
\hline
\end{tabular}

(Source: Atkin \& Wallack 1990: 16)

Adding to this, Tones \& Tilford (2010) suggests that 'unlike commercial companies which, after trying new brand images, abandon an unpopular product and produce something different that people do want, health promotion must continue to pursue its ethical and professional goals' (p. 347).

The fundamental objective underlying this process of information dissemination is to help the listener or the receiver to make informed choices. Gradually building the knowledge base of the population to make their individual health based decisions. Therefore it can be claimed that an effective health communication is to be tailored according to the needs of the listener/receiver. Research in this domain according to the various organizations should be concentrating on refining communication strategies to inform people about ways to enhance health or to avoid specific health risks.

As a discipline, health communication is still a branch of communication studies. As a powerful health communication strategy, media advocacy plays an important role in engaging people in dialogue about various aspects ofhealth promotion and disease prevention, facilitating community organizing to generate demand or support for health services, and potentially influencing policy-making on critical public health issues. Media channels, including print journalism, advertisements, fiction films, television shows, documentaries, and computer technology affect the 
healthcare system and individuals' use of that system (Friedman 2004).

Clearly, media representations of health and illness shape our understanding of the experience of illness, health, and healthcare and influence health beliefs, health behaviours, healthcare practices, and policy-making (Seale, 2004). Traditional media or other services used in the post-independence era were paid less attention to the kind of impact it had on health and health care services. In contrast, in them odernera, Information Technology revolution is said to have a drastic impact on health care empirically. There are numbers of literature signifying the growing usage and importance of ICT-enabled health care system. Considering this wide scope of health communication, scholars, practitioners, and policymakers recognize the significance of ICTenabled health communication to public health.

\section{New Age Communication: Information and Communication Technology}

Information procured especially from the ICT-enabled health care domain has transformed the way we look at illness/health on the whole.Earlier it was more like a centralized system of information flow containing of a pure bio-medical understanding which was far beyond a layman's ability to comprehend. But now technology has helped us gain a more holistic understanding of illness per say. This transformation has led to looking at patients more like consumers and doctors like service providers (Seale, 2004).

ICT is envisaged to play a critical role in improving the health care system through good quality services, sound communication systems followed by an efficient assessing system. By enlarge, it is often assumed that ICT is set out to eliminate disparities and discrepancies made on the basis of information. This complex system referred to as ICT in simple terms, encompasses a large portion of sub-systems within it apart from being the bedrock of innovation. It is also said to have left its imprints in the field of health. The recent breakthrough of Internet Revolution boosted the objective set out by ICT leading to a positive exploitation of all technology-based system of communication in order to improve 
the domain of health and health care. The base on which the efficiency of the health system is tested on is, how effectively it is responsive to the health needs of the population and its distributive pattern.

\section{Reviewing Health Communication}

Having discussed the definition and other nuances of health communication in this section we look at the ways scholars have understood and interpreted this whole discourse. This willbe done through highlighting some of the significant discussions and specific contributions from scholars already working in the field.

Health and illness discourses are pervasive in the print media, television, cinema, and the Internet (King and Watson 2005). The mass media in all its forms- print, television, radio and internet has an unparalleled reach as communication mechanisms. With $1 / 4^{\text {th }}$ internet user's 400 billion mobile users and other services, the United Nation Health Development Goal that is to 'connect' health care centers and hospitals with ICT by 2015 has now become a reality(Ramanan, 2015). Communication has now crossed all barriers which previously existed. It has substantial power in setting agendas. But there is no use of having sound technological services without people who can utilize them in an effective manner. In this context, it is of importance to know the audiences of health. Many scholars believe that the entire communication flow should be assessed based on them. This makes it important to understand little about the health audience in relation to media and how it affects their relation with the doctors. At the same time it also very interesting to see how many others consider the persuasive power of media as a threat. Health has often had the challenging task of both

- Using the media to influence health practices.

- Countering this same influence in situations where it encourages unhealthy choices.

These issues are especially acute in a crisis, such as (H1N1) 'swine' influenza pandemic. On such occasions hitting the right pitch is crucial, and difficult. Health communicators may need to advocate 
rapidly and effectively for the public adoption of basic preventive measures, like hand washing, while such messages may be displaced in a mass media dominated by discussion of technical interventions, such as thermal scanners (Thompson, Dorsey, Parrott, \& 2003, 2013). But at the same time to be more effective Henwood(2000) argues how media has been a delivery mechanism for getting the right information to the right people in the right way at the right time to promote personal change or awareness. Only then it can be a vehicle for increasing participation in the civic and political life and social capital to promote social change.

In today's media-rich landscape, and especially with the advancements of Information and Communication Technologies (ICTs), increasing efforts are underway to incorporate mass media strategies into health education, promotion, and disease prevention practices. At the same time, scholars have documented how mass media's reach to selective audiences has led to limited or moderate effects in influencing health knowledge, attitude, and behaviour.

To fully realize mass media's role in facilitating the pursuit of health education and promotion, and disease prevention health communicators need to exploit multiple mass media and interactive digital media channels and carry out carefully planned media strategies to reach intended audiences. Regardless of the medium or strategy Griffin (2001) has argued that three effects might follow:

- The learning of correct health information.

- The changing of health attitudes and values

- The establishment of new health behaviour

Most of the studies in this field use various kinds of tools and methods to find the role of media on health. One commonality is the similarity in their objectives, which were more or less coinciding with each other. The most important question to be answered was to analyze how information related to health is incorporated to serve the purpose of information dissemination to the right people, at the right time.Put in other words, how healthrelated messages are covered in a different medium to prove the 
involvement of media health communication fulfilling the already mentioned objective of increasing reachability.

For instance, Basavanthapa(2008) discusses how mass media should be used in combination with other methods for effective health communication and to bring changes in human behaviour in desired ways. The author talks about different types of mass media and the role they play in information dissemination. He gives a brief account of television, radio, newspapers, internet, mails, posters, billboards, exhibition, etc. According to the scholar, whatever may be the means of communication the public health communication methodologies must be culturally appropriate. Here Basavanthapa supports digital media to other forms of media for its success in reaching the masses and for the fact that it does not require articulating ability or supremacy over the language to understand the messages put forth. Taking into consideration the literacy rate of the country, the author's argument can be true to a large extent.

The article by Nab is quite relevant for the topic undertaken in this study. It not only gives us an insight into the new mediums of communication but also throws light on the significance of the same to the current health scenario. Here the author points out that currently, the social relationships between a doctor and patient is more of a discussion rather than a monologue. The 'one' authority to 'many' model has ceased to exist with the growth of newer technology. To give a better picture, he tries to pen the various new forms of ICT's like Twitter, Facebook, mobile phones, e-books, journals, etc. These has not just quickened the process of information dissemination but have widened the base of reliability and accessibility too (Nab, 2009).

$\mathrm{Nab}$ (2009) gives special attention to two significant concepts that are

- Credibility of information

- Ease of accessibility to information.

Both these concepts help us in understanding the potential of ICT in decision making. The major drawback of this new kind of revolution is the accuracy and credibility of information posted in 
all kinds of social media. This can form a new ground of study. Similarly, there is yet another issue which we dwelt upon in the previous paragraphs that is about whether the accessibility to the information is confined to a specific group of people or universally accessible. The bottom line of growing media and health communication is whether it is strategically and wisely used. If yes what, whom, when, and why, or is it a new form of traditional media focused on only 'passing down information.

The main role of media enabled communication is to pass down information. An article that touches the base or the fundamental aspects of media in terms of the role of media in disseminating health information is "Determinants of Infant and Child Mortality" (Gunashekaran, 2008).Here the author focuses on how mass media plays a pivotal role in propagating health information among the majority of the country that is the rural population. The contribution of this study lies in the way the author tries to show the significance of ICT-enabled communication systems in health care practices and other related fields of development.

Gunashekaran(2008) combines factual information regarding declining child or infant mortality rate to the intervention of contemporary communication systems. The study suggests that the decline in the mortality rate in certain pockets of India is minimal when compared to the child or infant mortality ratio at the global level. Nevertheless, the author admits that mass media interventions have definitely caused a change in the regular communication processes. The scholar has also emphasized on how the effective and efficient use of communication technology can bring about the desired change.

In contrast, another article written during the same period of time points out that in spite of the newer forms of technology which has emerged in recent times and the entire enthusiasm about ICT revolution, its usage is still minimal as it was in the past. In his book "Media for Health: Planning, Programmes, and Practice" the scholar authenticates his argument based on an empirical study made on media exposure and awareness about HIV/AIDS among the scheduled tribes of Nagaland and Chhattisgarh (Agrawal, 2009). Interestingly, in another study conducted at a similar time, where almost all the studied villages had access to radio and 
television, the analysis showed that the television was seen to be a much dominant source accepted as effective communication medium than the radio. This also means that the most useful mediums of communication as per the previous study, in which digital media were given prominence was proven to be not so significant in this community-based study(Basavanthapa, 2008). This illustration can be the best example to study partial information dissemination to a specific group. Based on the thorough analysis the results of the study showed that the human channels of communication that is spreading information among each other were said to be much swifter and clearer than the media enabled communication systems. This reason can be attributed to the characteristics of the tribal community that they are a smaller but closely knit group therefore though the results of this study cannot begeneralized. But the argument put forth by the author regarding usage of technology is a major concern of this project.

Another significant finding in the field of Health Communication is the presence of gender division. The findings of Agrawal (2009) reveals that men more than women in Nagaland and Chhattisgarh have been exposed to mass media. This means lot more new forms of communication which can reach all irrespective of difference can be considered as the need of the hour. As mentioned earlier interpersonal communication in the tribal regions are very strong since health workers have consistently been the source of information. Thus one can argue that if mass media as suggested by Basavanthapa (2008), can work along with different forms of interpersonal communication, i.e. spreading information through the community members, then it will be easier to popularize health related information. This argument is authenticated by the study done among the tribal population by Agrawal (2009), as it confirms this is a kind of chain effect where once a person gets the information it creates the need for more information or spreading the same. Thus the need for the hour is information dissemination, and this project believes that effective information dissemination is happening through ICT.

Another study tries and evaluates the effect of mother's exposure to ICT and use of prenatal care in India is by Gosh in which the results did not show any considerable impact of exposure top 
renatal care among mothers (Gosh, 2006). But, at the same time, the results of the study revealed the need for accessibility to electronic mediums or all forms of communication among the less privileged section of the population. This can be a new ground for study as to 'whether or not the new forms of ICT-enabled communication are nothing but new form of domination used by the elites against others?' The terminology which can comprehend this argument is 'scientific-bureaucracy' (Webster, 2007).

The author Ekzhawitz, in the book 'Capitalizing Knowledge' argues that innovation is an elitist view; therefore the new technologies are supported and created by the elite members of the society to retain the hegemonic influence over the rest. This not only leads us to think about the accessibility and deprivation aspect but also to inducing a new line of thought that is how these socalled haves act as opinion leaders in accepting and rejecting a new form of technology may it be in the medical field or the form of communication technology. Those with good ties in the world of networking and innovation is said to benefit out of this and others are isolated. This can not only be attributed to the illiterate, rural the economically deprived lot but this trend seems to be persistent even among the middle class (Ekzhawitz, 1998). This alsoforms a major ground for further study to identify and explore the categorizations within the umbrella term Middle-class and how they gain access to and effectively utilize Health Communication.

Based on the literature reviewed it can be said that there are very few works which have focused on the target group of health communication. The work of Giddens(1991) emphasizes on how situation influences audience's choice of resolving to information systems. They state that the audience may or may not seek health promoting information during high-risk moments. In fact, an individual's urge to resort to health promotional information is almost nil during such situations. Therefore their choice is limited when we study technology and health studies contextually. Studies conducted by various other renowned scholars like Hill(2000) who studied the 'reality television' followed by Langer(1998) brought out another aspect of audience, where they talk about what audience look for in a particular information, like emotional stimulation through dramatized contrasts that have an entertaining 
effect; fear and anxiety, for example, may be aroused so that they are experienced as a contrast to security and pleasure. The notion of the 'active audience' was designed to replace earlier conceptions of audiences as passive recipients of information, a conception often embedded in early health education studies of 'effects' (Giddens, 1990).

Going further in this line of debate, scholars have argued that people often relate themselves with the information they come across. In other words, there is a 'like me' syndrome when they come across information whether or not such information is actually related to them (Anderson, 1991). This is particularly important as this might not just lead to influential decisions made by the victims but also the ill effect of technological growth. It is said that 'with the very encounter of health-related information, people experience encounters of new diseases subconsciously. People draw on media to construct themselves - the media are mirrors for narcissistic experimentation with different potential selves' (Ibid). This kind research is quite relevant for this article as it makes us pay attention to the other side of media and its influence beyond the one envisaged. In the present scenario, where health communication is going beyond the control of the state and getting commercialized to suit the requirements of the private players in the field of health, it is essential and strongly recommended to understand the other side of health communication. The next article that we review completely ignores this dimension.

In an article titled "Establishing Private HealthcareFacilities in Developing Countries," the author Dawodu(2007) argues in favour of incorporating ICT in health communication. He draws his argument from the health care provider's point of view. According to this article, the three main players in the domain of health are the providers, practitioners, and consumers. The terminologies make it quite evident that the author builds his arguments keeping in mind the commercialization process in health care and how ICT is directly related to the reputation of the hospital. In the age of cutthroat competition, communication is the first brick laid for the growth of the private health care system. Therefore the marketing strategies used in the process of establishing a health care are of at 
most significance. The above-mentioned article becomes important to this study as it not only talks about the communication strategy from the provider's point of view but also emphasizes on how it is important to be updated about the impacts the strategies create.

Earlier the communication processes used in traditional media were more confined towards creating awareness, implementation of any programmes or policies. By and large, it was addressed just towards delivering the programmes planned by the state machinery or resolve the issue cropped up at that moment. However, today's communication strategies are more oriented towards preventive care, verification of services, products, etc., which can be seen as more preventive in nature. The direct consumer advertisements in the pharmaceutical industry are the best example which helps us understand how today's informed patients have been taking part actively in the decision-making process.

Nevertheless,Dawodu argues that one should not forget the contextual factors and other factors which inhibit the role of technology in mere information provision and nothing more (2007). When the patient is seriously ill and has little ability to compare the services provided, the cost-effectiveness of the service, decisions are left to others. Mostly in such critical situations, decision making is often left to the health care providers themselves. It is during such difficult situations the role of ICT can be put to the test, to see if it has guided the decisions of patients or their caretakers.

\section{Health Communication in Developing Countries: Issues and Possibilities}

In developed countries, the local interest groups often demand a higher level of accountability to show results for the use of the public funds whereas in developing countries the target communities usually have no voice in how funds are spent (Fox, 2012 , 55). The problem of measurement also has to do with the length of health communication programs in developing countries which go on for years (sometimes four to five years or more). Another drawback with health communication is that it cannot be 
replicated in a different context/society for a different set of target audience.

Capacity building is another important need for effective health communication in developing countries. History shows that so far most of the health communication activities in the developing countries are carried out with the help of the developed countries. 'As a result, public sector health communication departments in ministries of health and other government departments have been underfunded and understaffed and, consequently, have underperformed' (Fox, 2012, 63). In this context, we can take a more detailed account of India. For example, India's encounter with media revolution in the past decade: Like any other modern society mass media has become an important social institution in contemporary India. The different technologies used by mass media in India have undergone tremendous change as their reach has proliferated. Its content has been modified as the composition of the audience has also altered. Since their inception, all kinds of mass media have been playing important roles in the dissemination of information related to public health issues. For instance, in the initial phase Doordarshan the official channel of Indian Television used to focus mostly on Educational-Entertainment programs. The most innovative venture during this period was the first teleserial called HumLog which carried messages related to family planning, and education for the girl child. Since then television, along with other forms of mass media, has been deployed to spread awareness among the public regarding various social and health issues. These media are useful for their reach and acceptability even in remotest places. All these have raised the expectations from mass media. There is a greater demand for accurate, relevant, rapid and impartial information regarding public health and most importantly there is a growing reliance on mass media as the main source of information.

Although mass media is widely used to inform the public, there is very little data available regarding the involvement of public in the development and evaluation of these health-related communication. There are studies in India where the scholars have done a detailed content analysis of the programmes/campaigns that appeared in mass media (print and electronic media). To 
mention a few with which we are almost familiar are works done by Basavanthapa (1998), Gunasekaran (2008), Binod C. Agrawal (2009),T. M. Joseph (2007) and contributions of Debarchana Ghosh (2006). Their major objective (with few exceptions) is to study systematically how health-related messages are covered in different mediums to prove the involvement of mediain health communication. But their major limitations include less or no research on the urban scenario which excludes the urban poor as well; does not include any assessment of the new information communication and technology; does not include alternative perspectives to evaluate health communication in the age of privatization and commercialization of health care system. All these can be studied systematically in future to enrich the understanding of health communication in the age of Information revolution and privatization.

\section{Future Possibilities of Health Communication in India}

The punch line for Oxygen TV, launched by Oxygen Healthcare in Mumbai, speaks for itself: 'A Digital Revolution in Patient Education.' Oxygen TV is India's first and only captive audience network TV channel exclusively for doctor's waiting rooms and it focuses purely on health and lifestyle. Moreover, personalized health programmes featuring services for doctors and their patients, and embellished by personal messages/still photographs, herald a new beginning in the digital revolution. It is a network of specially designed LCD TVs placed at strategic touch points like Doctor's Waiting Room to deliver content focused on health and lifestyle, in infotainment and edutainment format. The strategic points in which the LCD TVs are placed ensure $100 \%$ viewership and aim to deliver a captive audience in an engaging and clutterfree environment. This canbe taken as one of the role models, and the same can be done on a massive scale to reach the people at the bottom line of the society.

Health communication can be more effective (especially in developing countries) if it provided with appropriate resources. For example, a proper campaign of Malaria eradication in a remote tribal region should be accompanied by free bed net distribution, teams with insecticides to spray in the houses, and the availability 
of effective drugs along with fairly modest investments in communication programs.

Research indicates that audience members who become more emotionally and psychologically involved with media personalities, both real celebrities, and fictional characters, are more likely to adopt the health beliefs and behavior modeled by those characters (Brown, 2012, p 137). Thus different types of popular culture like films, music and soap operas and the popular characters within these popular cultures (film stars, popular musicians, and singers, soap opera characters) can and should play a more prominent role in health communication.

Getting down to ICT, the Internet is one such area where our entire idea of communication has made a huge difference and in future can be improved upon. For instance, in order to deliver a crafted message to the public various innovative techniques are used which include promotional activities, advertisements or events. These not just helps draw the attention of the public but also passes information about the subject matter used in such activities.

The strategies help serve the dual goal of increasing demand for a product or a service and also draw attention towards the organizer thereby enhancing the brand name of the organization. For example, Acu-check is a company that has revolutionized the one touch diabetic checking programme by inventing or promoting a new diabetes checking equipment. The very first medium it used to publicize its product was the internet. The user turnover increased within no span of time. During this time there was a rampant spread of knowledge about diabetics as a serious illness. However, to enhance its turnover ratio the company tied up with hospitals providing free health based check-ups confining its target population to the age group falling between 40 and above. The people who accompanied members of this age group were usually their close relatives and friends. This helped spread the message and product in a more widespread manner. This is an example to show how innovative strategies can be used in the process of communicating information.

Other modes of communication would be providing free services, discounts on services/products, conferences, display boards, 
hoardings, screening videos, photos, etc. These examples are significant as their outreach depends mainly on the innovative usage of technology which determines the reachability of the message and also impacts it can create. One such innovative programmeis used by a Franco-Vietnamese Hospital, Malaysia. Other than the traditional modes of communication used to spread information the hospital also resorts to combining the medical card to an insurance programme which provides good quality services at affordable price to the consumers (Dawodu, 2007). In addition to benefiting an individual this programme has contributed by making early preventive medical care available to the consumers, the goal of any ICT-enabled services.

Similarly, Apollo is another large private health care provider who resorts to special strategies of communication to reach out to the public. Highlights of their innovative service are the usage of interactive web-based seminars, conferences, and information dissemination about the latest medical techniques used in the hospitals, etc. Apollo extensively relies on media and other established source of communication to instill confidence among the service takers. They have a special place among the patients by conducting events designed as Health Days, medical tourism packages to overseas patients, corporate stress management services, etc. This is a description to show the extensive reliance on the health care providers on ICT. Nevertheless the perception of the patients their immediate relatives and friends can still affect their decision of visiting a hospital. Similarly, the people who pay for the service or the health care providers themselves base their decision on the structural aspects of the hospitals. Taking all this into consideration, the communication strategy or marketing skills has an ample scope for study as it is seen to have a direct effect on the reputation of the hospital. The significance of this article to this study lies in the fact that this decision-making process is like a ripple effect or a chain process which flows between the three main players(doctors, patients and service providers) within the domain of health care.

One cannot disagree that sensitivity towards the patients' problems should be the main concern while resorting to any information communication services. But ICT follows a corporate culture even 
in health care system. Thus there is an urgent need to incorporate patient-centric approach in contemporary health communication systems which should be inculcated within this corporate culture. To do this, obtaining feedback from family and friends should be a significant part of the management system. It is to be understood that the patients usually vocalise their dissatisfaction to their close ones, but the inception of theonline feedback system and other rating systems have immensely been a source of reputation building and breaking. For example, Practo is an online application listing the top rated best hospitals and doctors available in a city. This rating is based on the feedback given by the patients after a particular consultation. Therefore a well-designed feedback system is necessary before it blemishes the reputation of the hospital. Along with Practo a few more health communication apps which are gaining significance and hence can become a subject for serious academic scrutiny are Ziffi, Lybrat, Continuous care Health App, Diabeto. As mentioned in the paper earlier these Apps which are meant for a specific kind of audience are quickly gaining popularity and with the increasing reach of other ICT mediums they are also going to be easily available to the masses. But their authenticity and effectiveness will remain a major question. A systematic study focusing on different dimensions of this new Information communication technology are the ground for future health communication research.

\section{Conclusion}

As final remarks to this paper, we can say that discussion on health communication carried out here highlights many significant ruptures in research in the contemporary scenario, and especially on India. To be more specific, there is ample scope to study the presence of new information communication technology in health communication and its impact on service providers, health practitioners, and the patients. It can be a highly significant and interesting study to analyze how the latest innovations in ICT have influenced the dynamics between the three stakeholders of health care. Similarly, a well-proposed research on Webster's scientific bureaucracy in the field of health communication can considerably 
enrich the process of knowledge production in the broader field of health and media.

\section{References}

Agrawal, Binod C (ed). (2009). Media for health: Planning and Practice. New Delhi, Concept Publishing Company.

Anderson, B. (1991). Imagined Communities:Reflections on the origin and spread of Nationalism. London: Verso.

Atkin, Charles \& Lawrence Wallack (ed). (1990). Mass Communication and Public Health: Complexities and Conflicts, New Delhi, Sage Publications.

Basavanthapa, BT. $(2008,1998)$. Community, Health, Nursing. New Delhi, Jaypee Brothers Medical Publishers (P) Ltd.

Brown, William J. (2012). 'Promoting Health through Entertainment-Education Media: Theory and Practice' in Obregon and Waisbord (ed) The Handbook of Global Health Communication. Malden, Oxford, Sussex, Wiley-Blackwell Publication, pp. 121-143.

Daniel Haluza M.D, D. j. (2014). 'ICT and the Future of Health Care: Aspects of Doctor-Patient Communication' inInternational Journal of Technology Assessment in Health Care, 298-305.

Fox, Elizabeth. (2012). 'Rethinking Health Communication in Aid and Development' in Obregon and Waisbord (ed) The Handbook of Global Health Communication. Malden, Oxford, Sussex, WileyBlackwell Publication, pp. 52-69.

Ghosh, Debarchana. (2006). 'Effects of Mother's Exposure to Electronic Mass Media on Knowledge and Use of Prenatal Care Service: A Comparative Analysis of Indian States' in The Professional Geographer, Oxford, Blackwell Publishing.

Giddens, A. (1990). The Consequences of Modernity. Cambridge: Polity Press.

Giddens, A. (1991). Modernity and Self Identity. Oxford: Polity.

Griffin, G. (2001). Representations of HIV and AIDS. Manchester: Manchester University Press.

Gunasekaran, S. (2008). Determinants of Infants and Child Mortality in Rural India. New Delhi, Kalpaz Publication. 
Joseph, T.M. (ed.) 2007. Local Governance in India: Ideas, Challenges, and Strategies. New Delhi, Concept Publishing Company.

Nab, C. M. (2009). Bulletin of the World Health Organization(August edition). Retrieved from Sci ELO Public health: http://dx.doi.org/ 10.1590/50042-968620090008000

Ramanan, D. K. (2015). Digitally Empowering. The Management Accountant, 22-26.

Seale, C. (2004). Health and the Media. Australia: Black well publishing.

Tones, Keith \& Sylvia Tilford. (2010). Health Promotion: Effectiveness, Efficiency, and Equity. New Delhi, Sage Publications.

Webster, A. (2007). Health, Technology and Society. London: Palgrave Macmillan.

WHO. (1948). WHO-Definition of Health. New York: International Health Conference.

Wyatt S Henwood. (2000). Technology and Internet Equality:Questioning the information society. London: Routledge publications. 